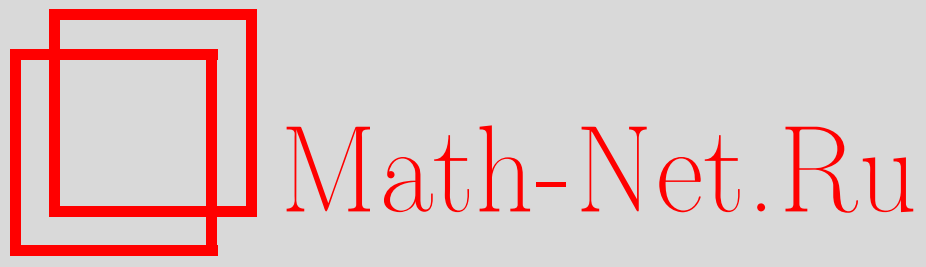

В. Е. Назайкинский, Б. Ю. Стернин, О принципе локальности индекса в эллиптической теории, Функи. анализ и его прил., 2001, том 35, выпуск 2, 37-52

DOI: https://doi.org/10.4213/faa244

Использование Общероссийского математического портала MathNet.Ru подразумевает, что вы прочитали и согласны с пользовательским соглашением

http://www.mathnet.ru/rus/agreement

Параметры загрузки:

IP : 3.82 .47 .9

26 апреля 2023 г., 14:21:33

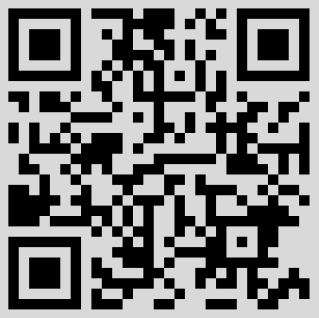


Функциональный анализ и его приложения

2001, т. 35, вып. 2, с. 37-52

УДК 517.9

\title{
О принципе локальности индекса в эллиптической теории*
}

\author{
(c) 2001. В. Е. НАЗАЙКИНСКИЙ, Б. Ю. СтЕРНИн
}

\section{Введение}

1. В последнее время в литературе появился ряд результатов, касающихся эллиптических операторов с условиями симметрии на многообразиях с особенностями. В число этих результатов входят, в частности, теоремы об индексе для общих эллиптических псевдодифференциальных операторов, а также для квантованных контактных преобразований (см., например, [1-5]). Основным средством для получения указанных результатов в этих статьях являлась подклейка второй копии многообразия и продолжение операторов на дубль. При этом дубль оказывался гладким замкнутым многообразием, для которого применима теория индекса Атьи-Зингера [6] (для случая псевдодифференциальных операторов) или теория Эпштейна-Мельроуза-Лайштнама-Неста-Цыгана $[7,8]$ (для случая квантованных канонических преобразований), а основная проблема состояла в том, чтобы выразить разность между удвоенным индексом первоначального оператора и индексом нового оператора на дубле в терминах инвариантов конормального символа. Подклеивание копии многообразия и продолжение оператора или, по крайней мере, символа на дубль (или, более общим образом, на многообразие, содержащее первоначальное многообразие в качестве части) является в действительности довольно старой идеей. В самом деле, эта техника была успешно применена к проблеме индекса краевых задач уже в довольно ранних работах советской математической школы по эллиптической теории (см., например, статьи Аграновича [9] и Дезина [10], а также обзор Аграновича [11], где можно найти более подробные ссылки) и позднее широко использовалась различными авторами. Мы упомянем здесь работы Хсианга и Стонга [12-14], где склейка с помощью обращающего ориентацию автоморфизма границы была применена для получения индекса оператора сигнатуры, а также работы Гилки и Смита $[15,16]$. Этот список, разумеется, можно продолжить.

2. Идеи продолжения на более широкое многообразие (и, в частности, подклеивание дубля) оказываются успешными в силу справедливости так называемого принциипа локальности индекса или теоремь об относительном индексе, общей трактовке и некоторым конкретным применениям которой и посвящена настоящая работа. Напомним, в чем заключается этот принцип.

Начнем с простейшей ситуации. Пусть $M-$ компактное гладкое многообразие без края, разделенное на две части $M_{-}$и $M_{+}$гладкой гиперповерхностью $S$ (рис. 1a). Предположим, что на $M$ задан эллиптический дифференциальный

*Работа выполнена при поддержке РФФИ, проекты 99-01-01100 и 00-01-00161, и Deutsche Forschungsgemeinschaft (1999). 


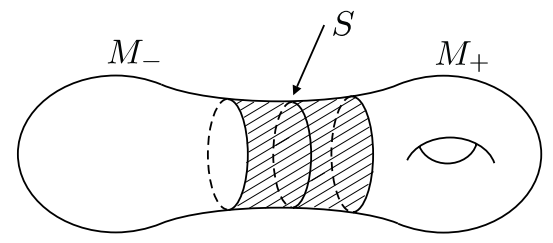

а) многообразие $M$

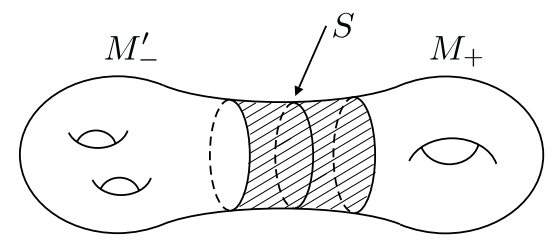

b) многообразие $M^{\prime}$

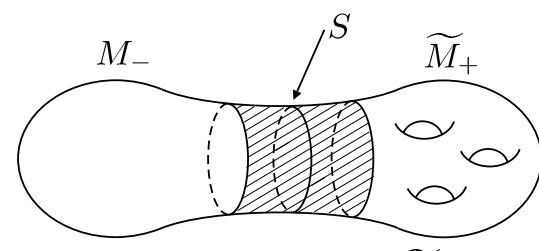

с) многообразие $\widetilde{M}$

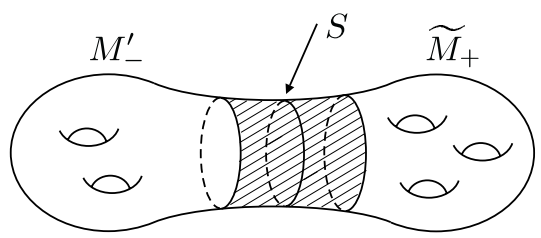

d) многообразие $\widetilde{M}^{\prime}$

Рис. 1. Простая перестройка

оператор $D$, действующий в сечениях некоторых векторных расслоений $E$ и $F$. Проделаем следующую операцию: «отрежем» $M_{-}$вдоль $S$ и приклеим к $M_{+}$другое многообразие $M_{-}^{\prime}$ так, чтобы $M_{-}^{\prime} \cup_{S} M_{+}$стало снова компактным гладким многообразием без края $M^{\prime}$ (рис. $1 \mathrm{~b}$ ). Разумеется, чтобы задать гладкую структуру при приклеивании, нужно на всех рассматриваемых многообразиях зафиксировать какую-либо тривиализацию воротниковой окрестности подмногообразия $S$ в виде прямого произведения. На рис. 1 такие окрестности $U \approx(-1,1) \times S$ заштрихованы. Далее, продолжим расслоения $\left.E\right|_{M_{+}}$и $\left.F\right|_{M_{+}}$до расслоений над всем $M^{\prime}$, а оператор $\left.D\right|_{M_{+}}$- до эллиптического оператора $D^{\prime}$ на всем $M^{\prime}$ в пространствах сечений этих новых расслоений. (Мы предполагаем, что такая операция возможна.) Разность

$$
\operatorname{ind}\left(D^{\prime}, D\right)=\operatorname{ind} D^{\prime}-\operatorname{ind} D
$$

индексов операторов $D^{\prime}$ и $D$ называется относительным индексом этих операторов. Зададимся теперь вопросом, зависит ли относительный индекс (0.1) от структуры операторов $D^{\prime}$ и $D$ на множестве $M_{+}$, где они совпадают. Более точно, проделаем такую же операцию отрезания и приклеивания с правой половиной многообразия, заменяя $M_{+}$на некоторую другую часть $\widetilde{M}_{+}$. Получим новые многообразия $\widetilde{M}$ и $\widetilde{M}^{\prime}$ (рис. 1c, d). Теперь, проделав соответствующие операции над расслоениями и операторами, мы приходим к новым операторам $\widetilde{D}$ и $\widetilde{D}^{\prime}$, которые по-прежнему совпадают между собой на правой половине многообразия, т. е. теперь на $\widetilde{M}_{+}$, а на левой половине совпадают соответственно с $D$ и $D^{\prime}$. Вопрос: будут ли совпадать относительные индексы этих двух пар операторов? Другими словами, будет ли иметь место равенство

$$
\operatorname{ind}\left(D^{\prime}, D\right)=\operatorname{ind}\left(\widetilde{D}^{\prime}, \widetilde{D}\right) ?
$$

Утвердительный ответ на этот вопрос тривиальным образом вытекает из так называемой «локальной формулы индекса» (см., например, [17]): индекс эллиптического оператора $D$ на замкнутом многообразии $M$ выражается формулой 


$$
\text { ind } D=\int_{M} \alpha(x)
$$

где «локальная плотность» $\alpha(x)$ в точке $x \in M$ зависит только от главного символа $\sigma(D)$ и его производных в слое над точкой $x$; остается лишь сравнить соответствующие интегралы для четырех случаев, показанных на рис. 1. (Такого рода рассуждение было использовано, например, в [18, Chap. 25].)

Равенство (0.2) и есть принцип локальности, или теорема об относительном индексе, для указанного выше простейшего случая. Однако его доказательство становится нетривиальным, если, по-прежнему предполагая, что $S$ компактно, мы откажемся от требования, чтобы $M$ было гладким компактным многообразием без края. В этом случае в нашем распоряжении уже нет формулы $(0.3)$, и известные доказательства принципа локальности (для некоторых частных случаев) опираются на методы, связанные с фундаментальным решением уравнения теплопроводности, или на формулу

$$
\text { ind } D=\operatorname{Trace}(1-R D)-\operatorname{Trace}(1-D R) \text {, }
$$

где $R$ - почти обратный к оператору $D$ с точностью до ядерных операторов. Метод, основанный на формуле $(0.4)$, был использован, например, в известной работе Громова и Лаусона [19] для доказательства теоремы об относительном индексе для операторов Дирака на полных некомпактных римановых многообразиях и вслед за тем в обобщении этой теоремы Анхелом [20] на случай произвольных существенно самосопряженных суперсимметричных фредгольмовых эллиптических операторов первого порядка. Эта же идея была использована и нами в предыдущих версиях данной работы $[21,22]$. Метод уравнения теплопроводности использовался в работе Доннели [23], где доказана теорема об относительном индексе для оператора сигнатуры, а также в работе Бунке [24]. Упомянем также книгу Бооса-Бавнбека и Войцеховского [18], а также работу Телемана [27], который, используя довольно тонкую гомотопическую технику, доказал теорему об относительном индексе (0.2) для операторов сигнатуры на липшицевых многообразиях, правда, лишь для случая, когда (в наших обозначениях) $M_{+}=\widetilde{M}_{+}$. В силу ограниченности объема статьи мы не можем дать исчерпывающий перечень результатов в этом круге вопросов. Заметим лишь, что известные теоремы Аграновича и Аграновича-Дынина $[9,25,26]$, выражающие относительный индекс пары краевых задач с одним и тем же краевым условием, но разными операторами (совпадающими на границе) или с одним и тем же оператором, но разными краевыми условиями, по сути дела представляют собой формулировку принципа локальности для краевых задач.

3. Техника подклеивания дубля успешно применялась и в теории индекса ПДО на многообразиях с особенностями. Теорема об индексе для операторов с симметричным конормальным символом была получена этим методом в [1]. Позднее идеи статьи [1] (вместе с использованием обращающего ориентацию диффеоморфизма границы) были применены в [3] для получения теоремы об индексе для операторов с условием симметрии, включающим указанный диффеоморфизм ${ }^{1)}$. Эту теорему можно применить к оператору Коши-Римана на двумерной поверхности, а также (как и в $[12,13])$ к оператору сигнатуры при условии, что база

1) K сожалению, авторам работы [3], по-видимому, не была известна более ранняя работа [12], где такая же склейка, как и в [3], была применена в ситуации многообразий с краем. 
конуса обладает упомянутым диффеоморфизмом. В [2] теорема об индексе для двумерных поверхностей получена в случае условия симметрии, включающего произвольный диффеоморфизм базы конуса. Отметим, что во всех приведенных выше результатах условие симметрии налагалось на полный конормальный символ.

Цель данной статьи заключается в том, чтобы доказать принцип локальности (0.2) в ситуации, достаточно общей, в частности, для того, чтобы его можно было применить как к эллиптическим ПДО, так и эллиптическим интегральным операторам Фурье на многообразиях с особенностями. Таким образом, речь идет о введении некоторого нового класса фредгольмовых операторов, для которого такой принцип локальности был бы справедлив и который включал бы как ПДО, так и интегральные операторы Фурье. Заметим, что класс абстрактных эллиптических операторов, введенный Атьей в [28] (и послуживший в дальнейшем отправной точкой для развития $K K$-теории) здесь непригоден, так как коммутаторы интегральных операторов Фурье с операторами умножения на функции, вообще говоря, не компактны.

Именно поэтому мы в $\$ 1$ вводим другой класс операторов и доказываем для него теорему об относительном индексе.

В §2 мы даем приложения к теоремам об индексе для ПДО и интегральных операторов Фурье на многообразиях с особенностями. Основное новшество в этих теоремах по сравнению с доказанными ранее в $[1,4,5]$ заключается в том, что условие симметрии, и это совершенно естественно, налагается только на главный символ конормального символа (а не на конормальный символ в целом). При этом вклад конических особых точек в индекс описывается с помощью спектрального потока семейства конормальных символов. Это понятие, близкое к понятию «потока дивизоров» из [29], обобщает введенное в [30] понятие спектрального потока семейства самосопряженных операторов.

4. Авторы признательны А. Ю. Савину и В. Е. Шаталову за полезные обсуждения и Б.-В. Шульце (ФРГ) за внимание к нашей работе и поддержку.

\section{§1. Пространства с перешейком и общий принцип локальности индекса}

1.1. Пространства с перешейком и эллиптические операторы. Цель этого раздела - определить следующие два важных понятия:

(а) абстрактный аналог понятия локализации сечения в $M_{-}, M_{+}$или воротниковой окрестности $U \approx(-1,1) \times S$, т. е. аналог понятия носителя сечения;

(b) абстрактный аналог понятия оператора, ядро Шварияа которого сосредоточено в окрестности диагонали.

ОПРЕДЕЛЕНИЕ 1.1. (а) Пространством с перешейком называется сепарабельное гильбертово пространство $H$, снабженное структурой модуля над коммутативной топологической алгеброй $C^{\infty}([-1,1])$ (действие непрерывно, единичная функция $1 \in C^{\infty}([-1,1])$ действует на $H$ как тождественный оператор).

(b) Носителем элемента $h \in H$ называется замкнутое множество

$$
\operatorname{supp} h=\bigcap \varphi^{-1}(0) \subset[-1,1],
$$

где $\varphi^{-1}(0)$ - полный прообраз точки 0 , а пересечение берется по всем таким элементам $\varphi \in C^{\infty}([-1,1])$, что $\varphi h=0$. Для произвольного подмножества $F \subset$ $[-1,1]$ через $H(F)$ будет обозначаться замыкание в $H$ множества всех элементов $h \in H$ с носителем в $F$. 
Поскольку в $C^{\infty}([-1,1])$ существует разбиение единицы, подчиненное любому локально конечному открытому покрытию, введенное таким образом понятие носителя обладает естественными свойствами. В частности, если $F_{1}, F_{2} \subset[-1,1]$ - такие подмножества, что $\bar{F}_{1} \cap \bar{F}_{2}=\varnothing$, то $H\left(F_{1} \cup F_{2}\right)=H\left(F_{1}\right) \oplus H\left(F_{2}\right)$ (сумма прямая, но не обязательно ортогональная).

ПримеР 1.2. Простейшим примером пространства с перешейком является гильбертово пространство

$$
H=L^{2}([-1,1] ; W)
$$

суммируемых с квадратом функций на отрезке $[0,1]$ со значениями в гильбертовом пространстве $W$. Произведение элемента $h \in H$ на элемент $\varphi \in C^{\infty}([-1,1])$ определяется естественным образом: $(\varphi h)(t)=\varphi(t) h(t)$.

Пример 1.3. Пусть $M-$ многообразие (возможно некомпактное, с особенностями и/или краем), и пусть $\chi: M \rightarrow[-1,1]-$ непрерывная функция, гладкая в гладкой части многообразия $M$ и такая, что $\overline{\chi^{-1}((-1,1))}-$ компактное множество, не содержащее особенностей многообразия $M$ и не пересекающееся с краем. Пространства Соболева $H^{s}(M)$ (в определении которых произвольным образом зафиксированы весовые функции вблизи особенностей и на бесконечности) естественно наделяются структурой пространств с перешейком: для $h \in H^{s}(M)$ и $\varphi \in C^{\infty}([-1,1])$ мы полагаем

$$
(\varphi h)(x)=\varphi(\chi(x)) h(x), \quad x \in M .
$$

Поскольку $\chi$ локально постоянна вне компактного множества, оператор умножения на $\varphi(\chi(x))$ непрерывен в $H^{s}(M)$ независимо от выбора веса. Если $M$ имеет непустой край, то естественная структура пространств с перешейком возникает и в пространствах Соболева $H^{s}(\partial M)$. Она задается той же формулой $(1.2)$, где на этот раз $h \in H^{s}(\partial M)$, а $x \in \partial M$.

Пример 1.4. Используя конструкцию из предыдущего примера, нетрудно снабдить пространства Соболева на каждом из многообразий, показанных на рис. 1, структурой пространств с перешейком. А именно, функция $\chi(x)$ выбирается таким образом, что она равна -1 слева от воротниковой окрестности $U$, равна 1 справа от $U$, а в $U$ она возрастает от -1 до 1 и зависит лишь от переменной $t$.

Конструкция, описанная в примере 1.3 , является основной в приложениях. Для однозначного задания этой конструкции достаточно, очевидно, задать конкретное многообразие $M$, пространства Соболева и функцию $\chi$.

ОПРеДЕлЕниЕ 1.5. Пусть $H_{1}, H_{2}-$ пространства с перешейками, а $F \subset[-1,1]$ - заданное подмножество. Мы говорим, что $H_{1}$ coвnaдaem на $F$ с $H_{2}$, и пишем $H_{1} \stackrel{F}{=} H_{2}$, если задан изоморфизм (не обязательно изометрический) $H_{1}(F) \approx$ $H_{2}(F)$. В этом случае мы также говорим, что $H_{1}$ и $H_{2}$ являются модификациями друг друга на $[-1,1] \backslash F$, и пишем $H_{1} \stackrel{[-1,1] \backslash F}{\longleftrightarrow} H_{2}$. Квадрат модификаций

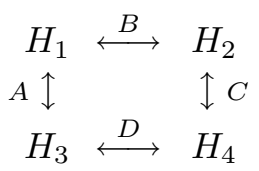


будем называть коммутативным, если коммутативна соответствующая диаграмма изоморфизмов

$$
\begin{array}{ccc}
H_{1}(F) & \approx & H_{2}(F) \\
\text { 孔 } & & \text { 亿 } \\
H_{3}(F) & \approx & H_{4}(F)
\end{array}
$$$$
F=[-1,1] \backslash(A \cup B \cup C \cup D) .
$$

Следующее определение формализует в контексте пространств с перешейком понятие оператора, который может быть прогомотопирован к оператору, ядро Шварца которого сосредоточено в произвольно узкой окрестности диагонали.

ОПРЕДЕЛЕНИЕ 1.6. (а) Правильным оператором в пространствах с перешейком $H, G$ называется непрерывное (в равномерной операторной топологии) семейство ограниченных линейных операторов

$$
D_{\delta}: H \rightarrow G, \quad \delta>0,
$$

удовлетворяющее следующему условию: для произвольного $\varepsilon>0$ существует $\delta_{0}>0$, такое, что

$$
\operatorname{supp} D_{\delta} h \subset U_{\varepsilon}(\operatorname{supp} h) \quad \forall h \in H
$$

при любом $\delta<\delta_{0}$. Здесь $U_{\varepsilon}(F)$ есть $\varepsilon$-окрестность множества $F$.

(b) Эллиптическим оператором в пространствах с перешейком $H, G$ называется правильный оператор (1.3), такой, что $D_{\delta}$ фредгольмов при каждом $\delta$ и имеет почти обратный $D_{\delta}^{[-1]}$, такой, что семейство $D_{\delta}^{[-1]}-$ правильный оператор.

Мы будем, как правило, опускать параметр $\delta$ в обозначении правильного оператора.

ЗАмЕчАНИЕ 1.7. Нетрудно видеть, что произведение правильных операторов (если оно определено) снова является правильным оператором. В пространствах с перешейком, полученных конструкцией из примера 1.3 на компактных многообразиях (возможно, с коническими особенностями) или многообразиях с краем, эллиптические псевдодифференциальные операторы или соответственно операторы эллиптических граничных задач являются эллиптическими в смысле приведенного выше определения (более точно, каждый такой оператор можно включить в семейство, зависящее от параметра $\delta$ и удовлетворяющее условиям определения). Это тривиальным образом следует из того, что псевдодифференциальные операторы и параметриксы граничных задач обладают свойством псевдолокальности. Умножая ядра этих операторов на семейство срезающих функций с носителем, стягивающимся к диагонали, получаем требуемые утверждения.

Пусть теперь

$$
A_{i}: H_{i} \rightarrow G_{i}, \quad i=1,2,
$$

- правильные операторы в пространствах с перешейком, и пусть $F \subset[-1,1]$ есть открытое подмножество (в топологии отрезка $[-1,1]$ ).

ОПреДЕЛЕНИЕ 1.8. МЫ говорим, что $A_{1}$ совпадает с $A_{2}$ на $F$ и пишем $A_{1} \stackrel{F}{=}$ $A_{2}$, если $H_{1} \stackrel{F}{=} H_{2}, G_{1} \stackrel{F}{=} G_{2}$ и для любого компакта $K \subset F$ выполнено следующее условие: существует $\delta_{0}>0$, такое, что

$$
A_{1 \delta} h=A_{2 \delta} h \quad \text { при } \delta<\delta_{0} \text { и } \operatorname{supp} h \subset K .
$$

(Это условие корректно, поскольку $h \in H_{1}(F) \approx H_{2}(F)$ и, так как множество $F$ открыто, $A_{1 \delta} h, A_{2 \delta} h \in G_{1}(F) \approx G_{2}(F)$ при малых $\delta$.) В этом случае мы 
говорим также, что $A_{1}$ получен из $A_{2}$ модификациией на $[-1,1] \backslash F$, и пишем $A_{1} \stackrel{[-1,1] \backslash F}{\longleftrightarrow} A_{2}$. Понятие коммутативного квадрата модификаций вводится очевидным образом.

Следующая лемма является важным (хотя и тривиальным) обобщением теоремы, утверждающей, что два почти обратных к фредгольмову оператору отличаются на компактный оператор.

ЛЕмма 1.9. Пусть $A_{1} \stackrel{F}{=} A_{2}$ - эллиптические операторы в пространствах с перешейком. Тогда $A_{1}^{[-1]} \stackrel{F}{=} A_{2}^{[-1]}+K$, где $K-$ правильныци компактный оператор. $B$ частности, при $A_{1}=A_{2}=A$ получаем, что любые два правильных почти обратных оператора $\kappa$ А отличаются на правильный компактный onepamop.

ДОКАЗАТЕЛЬСТВО. Пусть

$$
A_{1} A_{1}^{[-1]}=1+K_{1}, \quad A_{2}^{[-1]} A_{2}=1+K_{2},
$$

где $K_{1}, K_{2}$ - правильные компактные операторы. Тогда

$$
\begin{aligned}
& A_{2}^{[-1]} A_{1} A_{1}^{[-1]}=A_{2}^{[-1]}+A_{2}^{[-1]} K_{1}, \\
& A_{2}^{[-1]} A_{1} A_{1}^{[-1]} \stackrel{F}{=} A_{2}^{[-1]} A_{2} A_{1}^{[-1]}=A_{1}^{[-1]}+K_{2} A_{1}^{[-1]},
\end{aligned}
$$

откуда

$$
A_{2}^{[-1]} \stackrel{F}{=} A_{1}^{[-1]}+\left\{K_{2} A_{1}^{[-1]}-A_{2}^{[-1]} K_{1}\right\} .
$$

Доказательство завершено, так как оператор в фигурных скобках компактный.

ЛЕмма 1.10. Пусть $[-1,1]=\bigcup_{j} F_{j}$ - открылтое покрылтие $u A_{1} \stackrel{F_{j}}{=} A_{2}$ для всех $j$, где $A_{1}, A_{2}-$ правильные операторь. Тогда $A_{1 \delta}=A_{2 \delta}$ при достаточно мaлbıх $\delta$.

ДокАЗАТЕЛЬСтво. Возьмем конечное разбиение единицы, подчиненное покрытию $\left\{F_{j}\right\}$. Тогда утверждение леммы вытекает непосредственно из определения 1.8 .

\section{2. Общий принцип локальности (теорема об относительном индексе).} Теперь мы можем сформулировать и доказать основной результат - общий принцип локальности индекса.

ТЕОРемА 1.11. Пусть имеет место следующая коммутативная диаграмма модификаций эллиптических операторов в пространствах с перешейком:

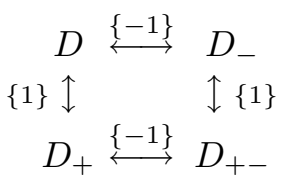

Tогда

$$
\operatorname{ind}(D)-\operatorname{ind}\left(D_{-}\right)=\operatorname{ind}\left(D_{+}\right)-\operatorname{ind}\left(D_{+-}\right) .
$$

ДОКАЗАТЕЛЬСтво. Введем обозначения для пространств, в которых действуют операторы, упомянутые в теореме:

$$
D: H \rightarrow G, \quad D_{-}: H_{-} \rightarrow G_{-}, \quad D_{+}: H_{+} \rightarrow G_{+}, \quad D_{+-}: H_{+-} \rightarrow G_{+-} .
$$


Тогда по определению имеются коммутативные диаграммы модификаций

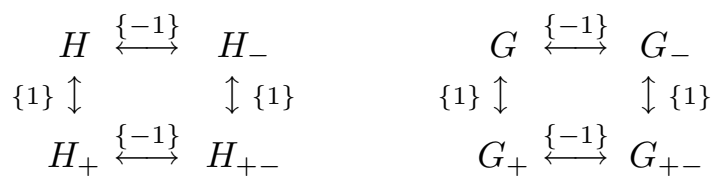

Лемма 1.12. Пусть диаграммы (1.6) тривиальнь, т.е. модификации тождественнь:

$$
H=H_{-}=H_{+}=H_{+-}, \quad G=G_{-}=G_{+}=G_{+-} .
$$

Тогда утверждение теоремы справедливо.

ДокАЗАТЕЛьство. Действительно, в этом случае

$$
\operatorname{ind}(D)-\operatorname{ind}\left(D_{-}\right)-\operatorname{ind}\left(D_{+}\right)+\operatorname{ind}\left(D_{+-}\right)=\operatorname{ind}\left(D D_{-}^{[-1]} D_{+-} D_{+}^{[-1]}\right) .
$$

Ho

$$
\begin{aligned}
& D D_{-}^{[-1]} D_{+-} D_{+}^{[-1]} \stackrel{(-1,1]}{=} D D^{[-1]} D_{+} D_{+}^{[-1]} \equiv 1, \\
& D D_{-}^{[-1]} D_{+-} D_{+}^{[-1]} \stackrel{\left[\frac{[-1,1)}{=}\right.}{=} D D_{-}^{[-1]} D_{-} D^{[-1]} \equiv D D^{[-1]} \equiv 1
\end{aligned}
$$

(где $\equiv$ означает равенство с точностью до правильных компактных операторов) и, следовательно,

$$
D D_{-}^{[-1]} D_{+-} D_{+}^{[-1]} \equiv 1
$$

по лемме 1.10 .

Теперь утверждение теоремы в общем случае вытекает из следующей леммы.

ЛЕмма 1.13. Изоморфизмы подпространств, входящих в определение модификаций в диаграммах (1.6), можно продолжить до изоморфизмов

$$
\begin{array}{cc}
H=H_{-} & G=G_{-} \\
\|=\| & \|\quad\| \\
H_{+}=H_{+-} & G_{+}=G_{+-}
\end{array}
$$

таким образом, итобы диаграммы (1.7) были коммутативны, а сами изоморфизмы коммутировали с новым действием алгебры $C^{\infty}([-1,1])$, задаваемьмм формулой

$$
\varphi * h=(\varphi \circ \chi) h
$$

где $\chi:[-1,1] \rightarrow[-1,1]-$ гладкая монотонная функция, которая равна -1 на $[-1,-1 / 2] u+1$ на $[1 / 2,1]$.

Действительно, относительно нового действия операторы $D, D_{-}, D_{+}, D_{+-}$ по-прежнему правильные и удовлетворяют нужной диаграмме модификаций, но действуют они теперь в одних и тех же пространствах, так что можно применить лемму 1.12 .

ДоКАЗАТЕЛЬСтво. Достаточно провести доказательство для первой диаграммы. Имеем разложения в прямую сумму

$$
\begin{array}{rlrl}
H & =H^{(-)} \oplus H((-1,1)) \oplus H^{(+)}, & H_{-} & =H_{-}^{(-)} \oplus H((-1,1)) \oplus H_{-}^{(+)}, \\
H_{+} & =H_{+}^{(-)} \oplus H((-1,1)) \oplus H_{+}^{(+)}, & H_{+-}=H_{+-}^{(-)} \oplus H((-1,1)) \oplus H_{+-}^{(+)},
\end{array}
$$


где, например,

$$
\begin{aligned}
& H^{(-)}=H([-1,-1 / 2)) \ominus(H([-1,-1 / 2)) \cap H((-1,1))), \\
& H^{(+)}=H([1 / 2,1)) \ominus(H([1 / 2,1)) \cap H((-1,1)))
\end{aligned}
$$

и т.д., а $A \ominus B$ обозначает ортогональное дополнение к $B$ в $A$.

Без ограничения общности можно считать, что пространства $H^{(-)}, H^{(+)}$и им подобные бесконечномерны (иначе можно добавить к ним прямые слагаемые вида $l^{2}$, а ко всем операторам - тождественные операторы id: $\left.l^{2} \rightarrow l^{2}\right)$. Далее, в силу диаграммы (1.6) имеем изоморфизмы

$$
H^{(+)}=H_{-}^{(+)}, \quad H_{+}^{(+)}=H_{+-}^{(+)}, \quad H^{(-)}=H_{+}^{(-)}, \quad H_{-}^{(-)}=H_{+-}^{(-)} .
$$

Остается произвольно выбрать изоморфизмы $H^{(-)} \approx H_{-}^{(-)}$и $H^{(+)} \approx H_{+}^{(+)}$; таковые всегда существуют между произвольными сепарабельными гильбертовыми пространствами. Полученные изоморфизмы (1.7) коммутируют с действием (1.8), поскольку $\varphi * h=\varphi(-1) h$ для элементов $h$ пространств $H^{(-)}, \ldots, H_{+-}^{(-)}$и $\varphi * h=$ $\varphi(1) h$ для элементов $h$ пространств $H^{(+)}, \ldots, H_{+-}^{(+)}$.

Лемма доказана.

ЗАмЕЧАНИЕ 1.14. Формула (0.2) является частным случаем доказанной теоремы. Это следует из конструкции примера 1.4 с учетом замечания 1.7.

\section{§2. Приложения к формулам индекса для операторов с условиями симметрии}

В этом параграфе мы даем приложения развитой выше теории к формулам индекса для операторов на многообразиях с коническими особенностями. Основные определения, касающиеся операторов на многообразиях с коническими особенностями, читатель может найти в [31]; там же можно найти дальнейшие ссылки.

\section{1. Теорема об индексе для псевдодифференциальных операторов на} многообразиях с коническими особенностями. Нам понадобится обобщение введенной в [32] версии понятия спектрального потока [30] на произвольные конормальные семейства.

1. Кратность особой точки операторного семейства. Пусть $H_{1,2}$ - гильбертовы пространства, а $D(p): H_{1} \rightarrow H_{2}-$ семейство ограниченных линейных операторов с параметром $p$, пробегающим открытое подмножество $\mathscr{U}$ комплексной плоскости. Будем говорить, что оно сильно конечномероморфно, если оно конечномероморфно и конечномероморфно обратимо (т. е. $D(p)$ мероморфно зависит от $p \in \mathscr{U}$ и главные части рядов Лорана в каждом из полюсов суть операторы конечного ранга; то же предполагается выполненным и для $\left.D^{-1}(p)\right)$. Ocoбымu точками семейства $D(p)$ называются полюсы семейства $D(p)$ и полюсы семейства $D^{-1}(p)$. Кратностью особой точки $p_{0}$ семейства $D(p)$ назовем число (всегда целое)

$$
m_{D}\left(p_{0}\right)=\text { Trace } \operatorname{Res}_{p=p_{0}}\left\{D^{-1}(p) \frac{\partial D(p)}{\partial p}\right\} .
$$

2. Определение спектрального потока семейства конормальных символов. Факты, изложенные в этом пункте, близко соприкасаются с результатами из [29]. 
Пусть $\Omega$ - гладкое компактное многообразие без края, и пусть

$$
D(p): H^{s}(\Omega, E) \rightarrow H^{s-m}(\Omega, F)
$$

- семейство ПДО порядка $m$ на $\Omega$, голоморфно зависящее от параметра $p \in \mathbb{C}$ и эллиптическое с параметром $p$ по Аграновичу-Вишику [33] в некотором двойном секторе ненулевого раствора, содержащем вещественную ось. Тогда это семейство является сильно конечномероморфным в указанном секторе. Такие семейства будем в дальнейшем называть конормальными символами на $\Omega$. Для простоты всюду далее, кроме примеров, предполагаем, что $m=0$.

Пусть $D_{t}=D_{t}(p)$ - непрерывно зависящее от параметра $t \in[0,1]$ семейство конормальных символов на $\Omega$. Мы хотим определить понятие спектрального потока sf $D_{t} \equiv \mathrm{sf}_{t=0,1} D_{t}$ семейства $D_{t}$. Интуитивное определение звучит следующим образом: sf $D_{t}$ - это алгебраическое число особых точек (с учетом кратности) семейства $D_{t}$, перешедших снизу вверх через вещественную ось (а точнее, из открытой нижней в замкнутую верхнюю полуплоскость) при изменении параметра $t$ от 0 до 1. Такое определение естественно обобщает понятие спектрального потока семейства $A_{t}$ нормально эллиптических операторов и переходит в него при $D_{t}=p-A_{t}$. Проблема, однако, заключается в том, чтобы дать строгое определение. В данном случае понятие спектрального сечения неизвестно (при $D_{t} \neq p-A_{t}$ ), и для определения спектрального потока мы воспользуемся иной, нежели в [32], конструкцией.

Предположим сначала (для упрощения формул), что при $t=0$ и 1 конормальный символ $D_{t}(p)$ не имеет особых точек на вещественной оси (это ограничение ниже будет снято). Разобьем отрезок $[0,1]$ на подынтервалы точками

$$
0=t_{0}<t_{1}<\cdots<t_{N}=1
$$

и выберем вещественные числа $\gamma_{i}, i=1, \ldots, N$, так, чтобы оператор $D_{t}(p)$ был обратим на весовой прямой $\operatorname{Im} p=\gamma_{i}$ при всех $t \in\left[t_{i-1}, t_{i}\right]$. При этом должно быть выполнено условие $\gamma_{1}=\gamma_{N}=0$. Существование такого разбиения и чисел вытекает из условия эллиптичности с параметром в секторе ненулевого раствора и непрерывной зависимости конормального символа от параметра $t$.

ОПрЕДЕлЕНИЕ 2.1. Спектральным потоком семейства $\left\{D_{t}\right\}_{t \in[0,1]}$ конормальных символов называется число

$$
\operatorname{sf} D_{t}=\sum_{i=1}^{N-1} g_{i}
$$

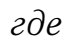

$$
g_{i}=\left\{\begin{array}{cl}
-\sum_{\operatorname{Im} p_{j} \in\left(\gamma_{i}, \gamma_{i+1}\right)} m_{D_{t_{i}}}\left(p_{j}\right), & \text { если } \gamma_{i}<\gamma_{i+1}, \\
\sum_{\operatorname{Im} p_{j} \in\left(\gamma_{i+1}, \gamma_{i}\right)} m_{D_{t_{i}}}\left(p_{j}\right), & \text { если } \gamma_{i} \geqslant \gamma_{i+1} .
\end{array}\right.
$$

Здесь $p_{j}$ - особые точки конормального символа $D_{t_{i}}$, попавшие в указанную полосу (заметим, что их конечное число, так что сумма определена корректно).

Теорема 2.2. Справедливы следующие утверждения:

(а) Oпределение 2.1 корректно (m.е. не зависит от выбора разбиения отрезка $[0,1]$ и чисел $\gamma_{j}$ ). 
(b) Спектральный поток sf $D_{t}$ зависит только от гомотопического класса пути $D_{t}$ (с фиксированньми началом и кониом). Более того, sf $D_{t}$ не изменяется и при таких деформациях (гомотопиях) пути $D_{t}$, что начало и конеи не фиксировань, но конормальные символь $D_{0}$ и $D_{1}$ не имеют особых точек на вещцественной оси ни при каком значении параметра гомотопии.

Теперь мы можем обобщить определение 2.1 на случай, когда $D_{0}(p)$ и $D_{1}(p)$ могут иметь особые точки на вещественной оси. Из условия эллиптичности с параметром вытекает, что существует такое $\varepsilon>0$, что в открытой полосе $-\varepsilon<\operatorname{Im} p<0$ нет особых точек конормальных символов $D_{0}(p)$ и $D_{1}(p)$. Рассмотрим семейство конормальных символов $D_{t, \tau}(p)=D_{t}(p-i \tau)$, зависящее от дополнительного вещественного параметра $\tau \in(0, \varepsilon)$. Тогда $D_{0, \tau}$ и $D_{1, \tau}$ не имеют особых точек на вещественной оси, и по теореме 2.2(b) спектральный поток sf $D_{t, \tau}$ не зависит от $\tau \in(0, \varepsilon)$. Мы полагаем по определению

$$
\text { sf } D_{t}=\operatorname{sf} D_{t, \varepsilon / 2} \text {. }
$$

\section{3. Связь спектрального потока с индексом.}

TЕOPЕMA 2.3. Пусть

$$
D_{t}(p): H^{s}(\Omega, E) \rightarrow H^{s}(\Omega, F), \quad t \in[0,1],
$$

- семейство конормальных символов на $\Omega$, причем $D_{0}(p)$ и $D_{1}(p)$ не имеют особых точек на вещественной оси. Пусть $\chi:(-\infty, \infty) \rightarrow[0,1], \chi(-\infty)=0$, $\chi(\infty)=1$, - гладкая функциия с экспоненциально убываюшими на бесконечности производньми. Положим

$$
D(\tau, p)=D_{\chi(\tau)}(p)
$$

и рассмотрим оператор

$$
\widehat{D} \stackrel{\text { def }}{=} D\left(\stackrel{2}{\tau},-i \frac{\partial}{\partial \tau}\right): H^{s}(C, E) \longrightarrow H^{s}(C, F)
$$

в пространствах Соболева ${ }^{1)}$ на бесконечном изилндре $C=\Omega \times(-\infty, \infty)$. Тогда

$$
\text { sf } D_{t}=- \text { ind } \widehat{D} \text {. }
$$

4. Теорема об индексе для эллиптических ПДО. Применим теперь полученные выше результаты для доказательства теоремы об индексе эллиптических ПДО на многообразиях с коническими особенностями. Эта теорема включает в себя ранее известные [1-3] как частные случаи.

Пусть $N$ - компактное многообразие без края с коническими особенностями (без ограничения общности считаем, что есть только одна коническая точка $\alpha$; база $\Omega$ соответствующего конуса не предполагается связной), и пусть

$$
\widehat{D}: H^{s}(N, E) \longrightarrow H^{s-m}(N, F)
$$

- эллиптический ПДО в пространствах Соболева на $N$ с конормальным символом $D_{0}(p)$.

1) Мы обозначаем распространения расслоений $E$ и $F$ на $C$ теми же буквами. Весовая прямая, входящая в определение пространств Соболева, есть $\{\operatorname{Im} p=0\}$, так что мы опускаем веса в обозначениях пространств и пишем $H^{s}$ вместо $H^{s, 0}$. 
Далее, пусть $g: \Omega \rightarrow \Omega-$ диффеоморфизм, а

$$
\mu_{E}:\left.E\right|_{\Omega} \longrightarrow g^{*}\left(\left.E\right|_{\Omega}\right), \quad \mu_{F}:\left.F\right|_{\Omega} \longrightarrow g^{*}\left(\left.F\right|_{\Omega}\right)
$$

- изоморфизмы расслоений. Предположим, что выполнено следующее условие.

УСловие А. Конормальные символь $D_{0}(p) u \mu_{F}^{-1} g^{*} D_{0}(-p)\left(g^{*}\right)^{-1} \mu_{E}$ гомотопны в классе конормальных символов ${ }^{1)}$.

Зафиксируем какую-либо гомотопию $D_{0 t}$,

$$
D_{00}(p)=D_{0}(p), \quad D_{01}(p)=\mu_{F}^{-1} g^{*} D_{0}(-p)\left(g^{*}\right)^{-1} \mu_{E} .
$$

Мы построим замкнутое многообразие $\mathscr{N}$ и расслоения $\mathscr{E}, \mathscr{F}$ над ним следующим образом. Выбрасывая из многообразия $N$ малую окрестность конической точки, получим многообразие $\widetilde{N}$ с краем $\partial \widetilde{N}=\Omega$. Подклеим два экземпляра многообразия $\widetilde{N}$ к двум торцам цилиндра $\Omega \times[0,1]$, используя для склейки тождественное отображение на левом торце и отображение $g$ на правом торце. Расслоения $\left.E\right|_{\Omega}$ и $\left.F\right|_{\Omega}$ распространим на цилиндр $\Omega \times[0,1]$ естественным образом и склеим их с соответствующими расслоениями на двух экземплярах многообразия $\widetilde{N}$ тождественным образом на левом торце и с помощью изоморфизмов $\mu_{E}, \mu_{F}$ на правом торце. Полученные расслоения над $\mathscr{N}$ обозначим через $\mathscr{E}$ и $\mathscr{F}$.

Теорема 2.4. При указанных условиях справедлива следующая формула индекса:

$$
\text { ind } \widehat{D}=\frac{1}{2}\left\{\text { ind } \mathscr{D}+\operatorname{sf} D_{0 t}\right\} \text {, }
$$

где $\mathscr{D}: H^{s}(\mathscr{N}, \mathscr{E}) \longrightarrow H^{s-m}(\mathscr{N}, \mathscr{F})$ - эллиптический оператор на замкнутом многообразии $\mathscr{N}$, который будет описан в доказательстве теоремы.

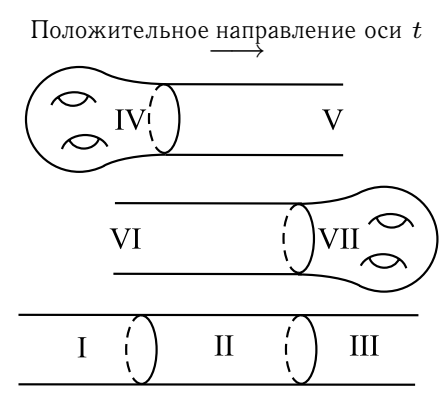

Исходная конфигурация
Положительное направление оси $t$

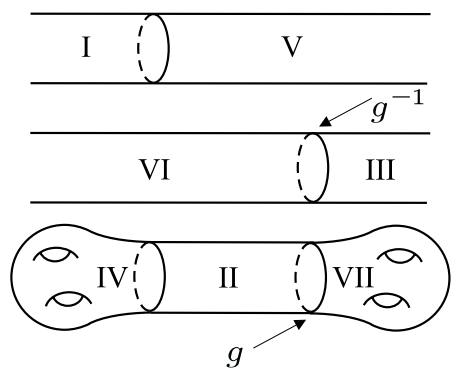

Конечная конфигурация

Рис. 2. Доказательство теоремы 2.4

ДоКАЗАТЕЛЬСтвО. Используя в окрестности конической точки замену переменной $r=e^{-t}$, где $r-$ расстояние до конической точки, мы можем представить $N$ как многообразие с цилиндрическим концом. Далее, оператор $D$ можно прогомотопировать в классе эллиптических ПДО, не меняя конормального символа, к оператору, коэффициенты которого не зависят от $t$ при достаточно больших $t$.

1) Условие А есть условие симметрии относительно точки $p=0$. Случай симметрии относительно точки $p \neq 0$ рассматривается аналогично. 
Будем считать, что это проделано с самого начала. Рассмотрим две конфигурации, показанные на рис. 2. Исходная конфигурация состоит из двух экземпляров многообразия $N$ с оператором $\widehat{D}$ и бесконечного цилиндра $(-\infty, \infty) \times \Omega$, на котором оператор задается с помощью операторнозначного символа $D_{0 \chi(t)}$, где функция $\chi:(-\infty, \infty) \rightarrow[0,1]$ возрастает от 0 до 1 и постоянна вне компактного множества. Конечная конфигурация состоит из многообразия $\mathscr{N}$ с оператором $\mathscr{D}$, главный символ которого совпадает с главным символом оператора $\widehat{D}$ на двух экземплярах многообразия $\mathscr{N}$ и с главным символом гомотопии $D_{0 t}$ на цилиндре $\Omega \times[0,1]$, и двух бесконечных цилиндров $(-\infty, \infty) \times \Omega$ с операторами $D_{0}(-i \partial / \partial t)$ и $D_{0}(i \partial / \partial t)$, коэффициенты которых не зависят от переменной $t \in(-\infty, \infty)$. Одна конфигурация получается из другой в результате перехода, аналогичного переходу от $M$ к $M^{\prime}$ на рис. 1, если через $M_{+}$обозначить объединение компонент I, III, IV, VII, а через $M_{-}$- всех остальных компонент. (Заметим, что $M_{-}^{\prime}$ отличается от $M_{-}$лишь расположением компонент.) Рассмотрим теперь новую часть $\widetilde{M}_{+}$, получающуюся из $M_{+}$заменой компонент IV и VII на полубесконечные цилиндры. (Новые операторы имеют не зависящие от $t$ коэффициенты на компонентах IV $+\mathrm{V}$ и VI+VII.) В новом варианте (отвечающем переходу от $\widetilde{M}$ к $\widetilde{M^{\prime}}$ на рис. 1) начальная и конечная конфигурация совпадают, так что относительный индекс равен нулю. Мы можем превратить пространства Соболева, в которых действуют операторы во всех указанных конфигурациях, в пространства с перешейком таким же образом, как это было проделано в примере 1.4 для простейших конфигураций, показанных на рис. 1. В соответствии с замечанием 1.7 можно считать, что все рассматриваемые в доказательстве операторы являются эллиптическими в смысле определения 1.6. Применяя теорему 1.11, получаем, что и в первом случае относительный индекс равен нулю, т. е. индекс для исходной и конечной конфигураций один и тот же, откуда, применяя теорему 2.3 , немедленно получаем утверждение доказываемой теоремы.

Для случая, когда гомотопия имеет вид $D_{0 \tau}(p)=B(p+i \tau), \tau \in\left[\gamma_{1}, \gamma_{2}\right]$, а отображение $g$ тождественное, получаем ранее известный результат из [1]. Используя результаты работы [34], легко перенести утверждение теоремы 2.4 на случай общих спектральных краевых задач с условием симметрии (теорема существования таких формул доказана в [32]).

\section{2. Теорема об индексе для интегральных операторов Фурье на много-} образиях с коническими особенностями. Задача о вычислении индекса интегральных операторов Фурье (более точно, квантованных контактных преобразований) была поставлена Вайнстейном $[36,37]$ и недавно решена (для операторов на гладких многообразиях) в $[7,8]$. В данном разделе мы сформулируем и докажем аналог теоремы 2.4 для интегральных операторов Фурье на многообразиях с коническими особенностями. Во избежание громоздкости ограничимся случаем, когда диффеоморфизм $g$, входящий в условие А, тождественный. Модификация наших рассуждений для случая нетривиального диффеоморфизма в условии симметрии не представляет трудности.

1. Интегральные операторы Фурье на многообразиях с особенностями. Дадим прежде всего определение интегральных операторов Фурье на многообразиях с коническими особенностями. Наше изложение будет по необходимости кратким. Более подробное изложение см. в $[4,5]$ (ср. также [35]). 
Пусть $N_{1}, N_{2}-$ многообразия с коническими особенностями, а $T^{*} N_{1}$ и $T^{*} N_{2}$ - кокасательные расслоения к $N_{1}$ и $N_{2}$, определяемые как сжатые кокасаmельные расслоения (compressed cotangent bundles) многообразий с краем $N_{1}^{\wedge}$ и $N_{2}^{\wedge}$, получаемых из $N_{1}$ и $N_{2}$ раздутием (blow-up) конических точек (см. [35]). Таким образом, многообразия $T^{*} N_{1}$ и $T^{*} N_{2}$ тоже суть многообразия с краем. Они снабжены естественной симплектической формой, имеющей особенность на крае; в конических координатах (см. [31]) форма имеет вид

$$
\omega^{2}=-\frac{d p \wedge d r}{r}+d q \wedge d \varphi
$$

где $\varphi-$ координата на базе конуса, $r-$ радиальная координата, $p-$ конормальная переменная, а $q-$ импульсная переменная, двойственная к $\varphi$.

Пусть

$$
g: T^{*} N_{1} \backslash\{0\} \rightarrow T^{*} N_{2} \backslash\{0\}
$$

- $\mathbf{R}_{+}$-однородное каноническое преобразование (т.е. отображение, гладкое вплоть до границы и сохраняющее симплектическую форму).

Пусть

$$
L(g)=\{(z, g(z))\} \subset T^{*} N_{1} \backslash\{0\} \times T^{*} N_{2} \backslash\{0\}
$$

- график отображения $g$; это множество является лагранжевым подмногообразием в $T^{*} N_{1} \backslash\{0\} \times T^{*} N_{2} \backslash\{0\}$ относительно симплектической формы $\pi_{2}^{*} \omega_{2}^{2}-\pi_{1}^{*} \omega_{1}^{2}$, где $\pi_{j}-$ проекция произведения на $j$-й сомножитель, а $\omega_{j}^{2}-$ симплектическая форма на $T^{*} N_{j}$.

Пусть $\Lambda$ - линейное расслоение Маслова над $L(g)$, а $a: L(g) \rightarrow \Lambda-$ не обращающееся в нуль сечение, однородное степени $m$.

Используя на $L(g)$ координаты, «снятые» с $T^{*} N_{1}$, можно считать $а$ сечением расслоения над $T^{*} N_{1}$. Мы определим интегральный оператор Фурье

$$
T(g, a)=H^{s, \gamma}\left(N_{1}\right) \rightarrow H^{s-m, \gamma}\left(N_{2}\right)
$$

станадартным образом как оператор с интегральным ядром $\mathscr{K}_{L_{g}}(a)$, где $\mathscr{K}_{L_{g}}$ - канонический оператор Маслова на $L_{g}$ (в его однородном варианте). Детали конструкции см. в $[4,5]$. При подходящем выборе элементов этой конструкции, конормальный символ $T_{0}(p)$ оператора $T(g, a)$ корректно определен как семейство операторов

$$
T_{0}(p): H^{s}\left(\Omega_{1}\right) \rightarrow H^{s-m}\left(\Omega_{2}\right),
$$

где $\Omega_{1}$ и $\Omega_{2}$ - базы конусов на многообразиях $N_{1}$ и $N_{2}$.

Оператор $T(g, a)$ будем называть эллиптическим на данной весовой прямой $\{\operatorname{Im} p=\gamma\}$, если конормальный символ $T_{0}(p)$ обратим всюду на весовой прямой.

2. Теорема об индексе. Введем следующее условие.

УсловиЕ В. Конормальные символь $T_{0}(p)$ и $T_{0}(-p)$ гомотопны в классе конормальных символов формально эллиптических интегральных операторов Фурье.

Теорема 2.5. Пусть $T(g, a)$ - эллиптический интегральный оператор Фурье, конормальный символ которого удовлетворяет условию В.

Тогда

$$
\text { ind } T(g, a)=\frac{1}{2}\left\{\text { ind } \mathscr{T}+\operatorname{sf} T_{0 t}\right\}
$$


где $T_{0 t}$ - гомотопия, связывающая $T_{0}(p)$ и $T_{0}(-p)$, a

$$
\mathscr{T}: H^{s}\left(\mathscr{N}_{1}\right) \rightarrow H^{s}\left(\mathscr{N}_{2}\right)
$$

- некоторый интегральный оператор Фурье на замкнутых многообразиях $\mathscr{N}_{1}$ и $\mathscr{N}_{2}$, получаемых из $N_{1}$ и $N_{2}$ той же конструкцией, что и $\mathscr{N}$ из $N$ в доказательстве теоремь 2.4.

Оператор $\mathscr{T}$, разумеется, получается из $T$ склейкой такого же рода, как и в теореме 2.4 .

Доказательство теоремы 2.5 воспроизводит доказательство теоремы 2.4 со следующими очевидными изменениями.

(1) Конструкция приклеивания дубля проводится отдельно для $N_{1}$ и $N_{2}$.

(2) Необходимо показать, что рассматриваемые интегральные операторы Фурье являются правильно эллиптическими, т.е. включить их в соответствующие семейства, зависящие от параметра $\delta$. Это нетрудно сделать, масштабируя цилиндрическую переменную $t$ на цилиндрических концах.

Выражение для индекса ind $\mathscr{T}$ интегрального оператора Фурье на паре замкнутых многообразий без края дается формулой Лейштнама-Неста-Цыгана [8], так что теорема 2.5 выражает индекс интегрального оператора Фурье на многообразии с коническими особенностями при условиях симметрии в терминах этой формулы и спектрального потока гомотопии конормальных символов.

\section{ЛИТЕРАТУРА}

1. Schulze B.-W., Sternin B., Shatalov $V$. On the index of differential operators on manifolds with conical singularities. Annals of Global Analysis and Geometry, 16, No. 2, 141-172 (1998).

2. Fedosov B. V., Schulze B.-W., Tarkhanov N. The index of higher order operators on singular surfaces. Pacific J. Math., 191, No. 1, 25-48 (1999).

3. Fedosov B. V., Schulze B.-W., Tarkhanov N. A Remark on the Index of Symmetric Operators. Univ. Potsdam, Institut für Mathematik, Potsdam, February 1998. Preprint $98 / 4$.

4. Nazaikinskii V., Schulze B.-W., Sternin B. The Index of Quantized Contact Transformations on Manifolds with Conical Singularities. Univ. Potsdam, Institut für Mathematik, Potsdam, August 1998. Preprint 98/16.

5. Назайкинский В. Е., Стернин Б. Ю., Шульце Б.-В. Индекс квантованных контактных преобразований на многообразиях с коническими особенностями. Докл. РАН, 368, № 5, 598-600 (1999).

6. Atiyah M. F. Singer I. M. The index of elliptic operators on compact manifolds. Bull. Amer. Math. Soc., 69, 422-433 (1963).

7. Epstein C., Melrose R. Contact degree and the index of Fourier integral operators. Math. Res. Lett., 5, No. 3, 363-381 (1998).

8. Leichtnam E., Nest R., Tsygan B. Local formula for the index of a Fourier integral operator. Preprint math.DG/0004022, 2000.

9. Агранович M. С. Эллиптические сингулярные интегродифференциальные операторы. УМН, 20, вып. 5, 3-20 (1965).

10. Дезин А. А. Инвариантные дифференциальные операторы и граничные задачи. Труды МИАН, т. 68, 1962.

11. Agranovich M. Elliptic boundary problems. In: Partial Differential Equations IX. Elliptic Boundary Value Problems (Agranovich M. S., Egorov Yu. V., Shubin M. A., eds.), Encyclopaedia of Mathematical Sciences, Vol. 79, Springer-Verlag, Berlin-Heidelberg, 1997, pp. 1-144.

12. Hsiung Ch.-Ch. The signature and $G$-signarute of manifolds with boundary. J. Differential Geom., 6, 595-598 (1972). 
13. Hsiung Ch.-Ch. A remark on cobordism of manifolds with boundary. Arch. Math., 27, 551-555 (1976).

14. Stong R. E. Manifolds with reflecting boundary. J. Differential Geom., 9, 465-474 (1974).

15. Gilkey P. B., Smith L. The eta invariant for a class of elliptic boundary value problems. Comm. Pure Appl. Math., 36, 85-132 (1983).

16. Gilkey P. B. Smith L. The twisted index problem for manifolds with boundary. J. Differential Geom., 18, No. 3, 393-444 (1983).

17. Gilkey P. B. Invariance Theory, the Heat Equation and the Atiyah-Singer Index Theorem. Publish of Perish. Inc., Wilmington Delawaere, 1984.

18. Booß-Baunbek B., Wojciechowski K. Elliptic Boundary Problems for Dirac Operators. Birkhäuser, Boston-Basel-Berlin, 1993.

19. Gromov M., Lawson H. B. Jr. Positive scalar curvature and the Dirac operator on complete Riemannian manifolds. Publ. Math. IHES, 58, 295-408 (1983).

20. Anghel $N$. An abstract index theorem on non-compact Riemannian manifolds. Houston J. of Math., 19, 1993, 223-237.

21. Назайкинский B. E., Стернин Б. Ю. Локализация и перестройки в теории индекса эллиптических операторов. Докл. РАН, 370, № 1, 19-23 (2000).

22. Nazaikinskii V., Sternin B. Surgery and the Relative Index in Elliptic Theory. Univ. Potsdam, Institut für Mathematik, Potsdam, Juli 1999, Preprint 99/17.

23. Donnelly H. Essential spectrum and the heat kernel. J. Funct. Anal., 75, 362-381 (1987).

24. Bunke U. Relative index theory. J. Funct. Anal., 105, 63-76 (1992).

25. Дьнин A. C. Многомерные эллиптические граничные задачи с одной неизвестной функцией. ДАН СССР, 141, 285-287 (1961).

26. Агранович М. С., Дьнин А. С. Общие краевые задачи для эллиптических систем в многомерной области. ДАН СССР, 146, 511-514 (1962).

27. Teleman $N$. The index of signature operators on Lipschitz manifolds. Publ. Math. IHES, 58, 39-78 (1984).

28. Atiyah M. F. Global theory of elliptic operators. In: Proc. of the Int. Symposium on Functional Analysis, University of Tokyo Press, Tokyo, 1969, pp. 21-30.

29. Melrose $R$. The eta invariant of pseudodifferential operators and families. Math. Res. Lett., 2, No. 5, 541-561 (1995).

30. Atiyah M., Patodi V., Singer I. Spectral asymmetry and Riemannian geometry III. Math. Proc. Cambridge Philos. Soc., 79, 71-99 (1976).

31. Schulze B.-W., Sternin B., Shatalov V. Differential Equations on Singular Manifolds. Semiclassical Theory and Operator Algebras, Mathematics Topics, Vol. 15, Wiley-VCH Verlag, Berlin-New York, 1998.

32. Савин А. Ю., Стернин Б. Ю., Шульце Б.-В. Об инвариантных формулах индекса спектральных краевых задач. Дифференциальные уравнения, 35, № 5, 705-714 (1999).

33. Агранович М. С., Вишик М. И. Эллиптические задачи с параметром и параболические задачи общего вида. УМН, 19, вып. 3, 53-161 (1964).

34. Назайкинский В. Е., Стернин Б. Ю., Шаталов В. Е., Шульце Б.-В. Спектральные краевые задачи и эллиптические уравнения на многообразиях с особенностями. Дифференциальные уравнения, 34, № 5, 695-708 (1998).

35. Melrose R. Transformation of boundary problems. Acta Math., 147, 149-236 (1981).

36. Weinstein $A$. Fourier integral operators, quantization, and the spectra of a Riemannian manifolds. In: Géométrie Symplectique et Physique Mathématique, Colloque Internationale de Centre National de la Recherche Scientifique, Vol. 237, 1975, pp. 289-298.

37. Weinstein $A$. Some questions about the index of quantized contact transformations. RIMS Kôkûryuku, 104, 1-14 (1977). 